\title{
A Selective Toxicity Toward Cultured Mesencephalic Dopaminergic Neurons Is Induced by the Synergistic Effects of Energetic Metabolism Impairment and NMDA Receptor Activation
}

\author{
Isabel Marey-Semper, Michèle Gelman, and Matthieu Lévi-Strauss \\ Chaire de Neuropharmacologie and INSERM U 114, Collège de France, 75231 Paris cedex 05, France
}

Numerous observations strongly support the hypothesis that dopaminergic neurons could be particularly vulnerable to an impairment of their energetic metabolism. In order to demonstrate the existence of such a selective vulnerability, the toxic effects of rotenone, an inhibitor of complex I of the respiratory chain, and of glutamate, which is very likely involved in the neurotoxicity induced by an energetic stress, were analyzed on cultured mouse mesencephalic neurons. Toxicity toward dopaminergic and GABAergic neurons was compared by measuring the residual uptakes of dopamine and GABA. Exposure to $5 \mathrm{~nm}$ rotenone for 6 $\mathrm{hr}$ or to a low concentration of glutamate $(100 \mu \mathrm{M})$ for $1 \mathrm{hr}$ did not lead to a high selective toxic effect on dopaminergic neurons. In contrast, dopaminergic neurons were three times less resistant to the sequential exposure to rotenone and glutamate than GABAergic neurons. A particular resistance of mesencephalic GABAergic neurons to the synergistic toxic effects of rotenone and glutamate was ruled out since two other neuronal types, the striatal cholinergic and GABAergic neurons, displayed the same weak vulnerability as the mesencephalic GABAergic neurons. This selective toxic effect of glutamate on rotenone-pretreated dopaminergic neurons was blocked by either AMPA or NMDA receptor antagonists and mimicked by combined treatment with AMPA and NMDA, or by NMDA alone when the medium was deprived of $\mathrm{Mg}^{2+}$ ions. Moreover, this NMDA-selective neurotoxicity was critically dependent on the presence of a physiological extracellular sodium concentration, since the use of choline chloride instead of sodium chloride had a protective effect on dopaminergic neurons. Our results indicate that both the activation of NMDA receptors and the impairment of the energetic metabolism induce a selective toxicity toward mesencephalic dopaminergic neurons. This could therefore explain their natural degeneration in the course of Parkinson's disease, in which mitochondrial abnormalities have been recently described.

[Key words: Parkinson, dopamine, rotenone, glutamate,

Received Feh. 22, 1995; revised Apr. 24, 1995; accepted Apr. 27, 1995.

Professor Jacques Glowinski is gratefully acknowledged for his support during this study. We thank Drs. Philippe Marin, Nephi Stella, and Robert Williams (Collège de France, Paris) for fruitful discussions and critical reading of the manuscript. We thank also Dr. André Chéramy for his kind help in HPLC determination of glutamate concentrations. This work was supported by the Bioavenir programme (Rhône-Poulenc Rorer S.A., Ministère de la Recherche et de l'Espace and Ministère de l'Industrie et du Commerce Extérieur).

Correspondence should be addressed to Isabel Marey-Semper, Chaire de Neuropharmacologie and INSERM U114, Collège de France, 11, Place Marcelin Berthelot, 75231 Paris cedex 05, France.

Copyright (C) 1995 Society for Neuroscience $0270-6474 / 95 / 155912-07 \$ 05.00 / 0$
NMDA receptor, neurotoxicity, mesencephalic neurons, mitochondria]

The selective vulnerability of a particular subset of neurons is a common feature of neurodegenerative diseases such as Parkinson's disease, Huntington's disease, and amyotrophic lateral sclerosis. Parkinson's disease is mainly characterized by the degeneration of the nigrostriatal dopaminergic neurons. Numerous data suggest an involvement of the energetic metabolism in the selective vulnerability of these cells. Widespread mitochondrial abnormalities have indeed been described in Parkinsonian patients, suggesting that this disease could be due to some alterations of oxidative phosphorylation (for review, see Di Mauro, 1993). Moreover, the nigrostriatal dopaminergic neurons are selectively destroyed in mouse (Heikkila et al., 1984), monkey (Langston et al., 1984), and human (Davis ct al., 1979) following the systemic administration of 1-methyl-4-phenyl-1,2,3,6 tetrahydropyridine (MPTP), an inhibitor of the complex I of the respiratory chain, which is less toxic toward the other catecholaminergic neurons in spite of their ability to accumulate it. Similarly, the local injection of rotenone, an inhibitor of the complex I of the respiratory chain, into the median forebrain bundle greatly diminished the striatal content of dopamine without affecting that of GABA (Heikkila et al., 1985). This latter result suggests that rotenone is more toxic for the nigrostriatal dopaminergic neurons than for the striatonigral GABAergic neurons. Previously, we have proposed that a metabolic weakness of the dopaminergic neurons could account for their particular vulnerability to impairment of energetic metabolism (Marey-Semper et al., 1993). In order to substantiate this hypothesis, we had compared, as an index of the energetic metabolism, the inhibitory effects of rotenone on the high-affinity uptake of different neurotransmitters in mouse striatal synaptosomes or cultured mesencephalic neurons. In both preparations, the uptake of dopamine was much more affected than that of other neurotransmitters, suggesting indeed that intrinsic metabolic properties of the dopaminergic neurons could explain their natural vulnerability (Marey-Semper et al., 1993).

In the present study, to further assess the existence of a greater vulnerability of dopaminergic neurons to an energetic stress, we have compared the sensitivity of cultured murine mesencephalic neurons to the neurotoxic effects of rotenone. Since neuronal vulnerability following a metabolic impairment appears to be related to glutamate receptor activation (Novelli et al., 1987; Zeevalk et al., 1991, 1995), we also investigated the toxic effects of this excitatory amino acid.

Cultured dopaminergic neurons were found to be only slightly 
more sensitive than GABAergic neurons to the toxic effects of either rotenone or glutamate. However, these compounds, when used together, were surprisingly much more toxic for dopaminergic neurons than for GABAergic neurons.

\section{Materials and Methods}

Biochemicals reagents. All biochemical reagents were purchased from Sigma except when indicated.

Neurotransmitter uptake measurements in cultured neurons. Dissociated mesencephalic neurons from 14-d-old Swiss mouse embryos were prepared as previously described (Chamak and Prochiantz, 1989) with the following modifications. Culture dishes were incubated at $37^{\circ} \mathrm{C}$ for $3 \mathrm{hr}$ in Dulbecco's modified Eagle's medium (DMEM) (Gibco) supplemented with $10 \%(\mathrm{v} / \mathrm{v})$ fetal calf serum. Wells were washed and cells were plated at a density of $0.2510^{6}$ per $\mathrm{cm}^{2}$ in 24- or 96-well plates in DMEM medium supplemented with D-glucose ( $33 \mathrm{~mm}$ ) (Merck), L-glutamine (2 mM) (Gibco), $\mathrm{NaIICO}_{3}(3 \mathrm{~mm})$ (Merck), HEPES ( $5 \mathrm{~mm}$, pH 7.4) (Gibco), $0.1 \%$ ovalbumin ( $w / v)$, insulin $(25 \mu \mathrm{g} / \mathrm{ml})$, iron-free transferrin $(100 \mu \mathrm{g} / \mathrm{mI})$, progesterone $(20 \mathrm{nM})$, putrescin $(60 \mu \mathrm{M})$, and selenium salt $(30 \mathrm{~nm})$. After $5 \mathrm{~d}$, the culture medium was replaced by fresh medium containing either rotenone dissolved in DMSO or DMSO alone $(1 / 200, \mathrm{v} / \mathrm{v})$. After a $6 \mathrm{hr}$ incubation period, L-glutamic acid $(100$ $\mu \mathrm{M}$ final concentration), AMPA (100 $\mu \mathrm{M}$, Tocris Neuramin), or NMDA $(200 \mu \mathrm{M})$ was added for $1 \mathrm{hr} .(+) \mathrm{MK} 801(1 \mu \mathrm{M})$ was added $5 \mathrm{~min}$ prior to glutamate agonists, whereas D(-)APV (500 $\mu \mathrm{M}$, Research Biochemical International) and DNQX (50 $\mu \mathrm{M}$, Research Biochemical International) were added at the same time as the glutamate agonists. In some experiments (see Table 3, Fig. 3), cells were washed once with Krebs bicarbonate buffer $(124 \mathrm{~mm} \mathrm{NaCl}$ or $124 \mathrm{~mm}$ choline chloride, $35 \mathrm{~mm}$ $\mathrm{KCl}, 1.25 \mathrm{mM} \mathrm{K}_{2} \mathrm{HPO}_{4}, 26.3 \mathrm{mM} \mathrm{NaHCO}, 1.2 \mathrm{mM} \mathrm{CaCl}, 10 \mathrm{~mm}$ glucose) and incubated for $1 \mathrm{hr}$ in the same buffer with or without $\mathrm{MgSO}_{4}(1.2 \mathrm{mM})$, AMPA $(100 \mu \mathrm{M})$, NMDA (200 $\left.\mu \mathrm{M}\right)$, and tetrodotoxin (TTX) $(1 \mu \mathrm{M})$. In all cases, cells were washed twice and further incubated in fresh DMEM medium for 16-17 hr.

After this $16-17 \mathrm{hr}$ incubation period, cells were placed on ice, washed once with cold phosphate-buffered saline, $\mathrm{pH} 7.4$, containing glucose $(5 \mathrm{~mm})$ and $\mathrm{Ca}^{2+}-\mathrm{Mg}^{2+}$ (PBS-glucose). Uptake measurements were performed by incubating neurons at $37^{\circ} \mathrm{C}$ for $15 \mathrm{~min}$ in $\mathrm{PBS}$ glucose supplemented with: dopamine (DA) uptake (1 mM ascorbic acid, $30 \mathrm{~nm}$ DA, $0.9 \mu \mathrm{Ci} / \mathrm{ml})$; GABA uptake $(10 \mu \mathrm{M} \mathrm{GABA}, 2.5 \mu \mathrm{Ci} /$ $\mathrm{ml})$; serotonin $(5-\mathrm{HT})$ uptake $(100 \mathrm{~nm} 5-\mathrm{HT}, 1 \mu \mathrm{Ci} / \mathrm{ml})$; choline uptake [40 nM choline, $3.25 \mu \mathrm{Ci} / \mathrm{ml}\left({ }^{3} \mathrm{H}-\mathrm{DA} 36 \mathrm{Ci} / \mathrm{mmol} \mathrm{NEN}\right.$, and ${ }^{3} \mathrm{H}-\mathrm{GABA}$ $94 \mathrm{Ci} / \mathrm{mmol},{ }^{3} \mathrm{H}-5-\mathrm{HT} 10.4 \mathrm{Ci} / \mathrm{mmol},{ }^{3} \mathrm{H}$-choline $81 \mathrm{Ci} / \mathrm{mmol}$, Amersham)]. For GABA uptake, incubation medium was supplemented with $\beta$-alanine $(500 \mu \mathrm{M})$ to prevent astrocytic GABA uptake (Schousboe et al., 1979). The reaction was stopped by placing cells on ice and by two washes with ice-cold PBS-glucose. Neurons were lysed with a $1 \%$ sodium dodecyl sulfate (SDS) solution $(\mathrm{w} / \mathrm{v}$ ) and radioactivity was measured by liquid scintillation counting. Nonspecific uptake values were determined in the presence of benztropine $(1 \mu \mathrm{M}$, Merck), nipecotic acid $(500 \mu \mathrm{M})$, or imipramine $(1 \mu \mathrm{M})$, which are specific inhibitors of DA, GABA, and 5-HT uptakes, respectively (Coyle et al., 1969; Javaïd et al., 1979; Schousboe et al., 1979). For choline, nonspecific uptake was measured at $0^{\circ} \mathrm{C}$. The mean of nonspecific uptake values was subtracted from each determination. Results are expressed in percent of control values obtained in the absence of rotenone and glutamate. Each determination was carried out in either triplicate or quadruplicate.

Differential toxicity ratio (DTR) value. In order to quantify the degree of selectivity toward dopaminergic neurons of the toxicity induced on cultured mesencephalic neurons by various treatments, we used the following ratio:

(GABA uptake, \% of control) - (DA uptake, \% of control)

$$
\text { (GABA uptake, \% of control) }
$$

defined as the differential toxicity ratio (DTR).

Assay of cell survival. Cell survival was estimated with the 3-[4,5 dimethylthiazol-2-yl]-2,5-diphenyl-2H-tetrazolium bromide (MTT, Merck)based colorimetric assay (Denizot and Lang, 1986). Briefly, cells were incubated for $3 \mathrm{hr}$ at $37^{\circ} \mathrm{C}$ in the presence of MTT $(0.5 \mathrm{mg} / \mathrm{ml})$ dissolved in fresh DMEM medium. The incubation medium was replaced by DMSO and the absorption at $570 \mathrm{~nm}$ was measured. Results were expressed in percent of control values obtained in the absence of rotenone and glutamate.

Anti-tyrosine hydroxylase immunocytochemistry. Cells were fixed with $4 \%$ PBS-formaldehyde for $20 \mathrm{~min}$ at room temperature. After two washes with PBS containing bovine serum albumin $5 \%(\mathrm{w} / \mathrm{v})$ and tween $0.1 \%(\mathrm{v} / \mathrm{v})$ (PBS-BSA-T), cells were incubated for $2 \mathrm{hr}$ with mouse monoclonal anti-tyrosine hydroxylase antibody ( $40 \mu \mathrm{g} / \mathrm{ml}$, Boehringer) diluted 25-fold in PBS-BSA-T at room temperature. Cells were then washed twice with PBS-BSA-T and incubated for $1 \mathrm{hr}$ with peroxidasecoupled goat anti-mouse IgG (1 mg/ml, BioSys) diluted 100-fold in PBS-BSA-T. After two washes with PBS-BSA-T, cells were rinsed with Tris (100 mM, pH 7.5). Peroxidase activity was revealed by incubation with $3-3^{\prime}$-diaminobenzidine $[0.04 \%(\mathrm{w} / \mathrm{v})]$ and $\mathrm{H}_{2} \mathrm{O}_{2}$ diluted $[0.02 \%(\mathrm{v} /$ v)] in Tris (100 mM pH 7.5) for 6-8 min. The rcaction was stopped by rinsing wells with water. The number of stained neurons bearing at least one neurite was determined in each well by counting 24 microscopic fields covering $5.3 \%$ of the well surface. Results are expressed in percent of control values obtained in the absence of rotenone and glutamate.

\section{Results}

Effects of rotenone and glutamate on mesencephalic dopaminergic and GABAergic neurons

In order to establish whether dopaminergic neurons displayed a greater vulnerability than GABAergic neurons to metabolic stress, the loxic effect of rotenone on cultured mesencephalic neurons from 14-d-old mouse embryos was analyzed. Rotenone was chosen as a metabolic inhibitor of the complex $\mathrm{I}$ of the respiratory chain (Ernster et al., 1963) because its lipophilic character suggests that it can penetrate into all neuronal types with equivalent efficiency. The delayed toxicity induced by a 6 $\mathrm{hr}$ rotenone exposure on mesencephalic dopaminergic and GABAergic neurons was estimated by measuring, after a $16 \mathrm{hr}$ recovery period, the residual high-affinity uptakes of DA and $\mathrm{G} \Lambda \mathrm{B} \Lambda$ used as indices of viability of the dopaminergic and $\mathrm{G} \Lambda$ BAergic (Radian et al., 1990) neurons.

Rotenone concentrations lower than $0.5 \mathrm{~nm}$ did not induce any decrease in neurotransmitter uptake, whereas both uptake values reached background levels for rotenone concentrations higher than $10 \mathrm{~nm}$ (data not shown). The application of $5 \mathrm{~nm}$ rotenone resulted in a residual specific uptake of $43 \pm 3 \%$ for $\mathrm{DA}$ and $48.5 \pm 3 \%$ for GABA (Fig. $1 \mathrm{~A}$ ), suggesting that rotenone does not exert a selective neurotoxicity on dopaminergic neurons.

Mesencephalic dopaminergic and GABAergic neurons were then exposed for $6 \mathrm{hr}$ to various concentrations of rotenone and for $1 \mathrm{hr}$ to $100 \mu \mathrm{M}$ glutamate, the neurotoxicity of these treatments being estimated as before following a $16 \mathrm{hr}$ recovery period. Dopaminergic neurons were found to be three times more vulnerable than GABAergic neurons to the prolonged exposure to $5 \mathrm{nM}$ rotenone followed by the $1 \mathrm{hr}$ application of $100 \mu \mathrm{M}$ glutamate (Fig. 2). This higher vulnerability of the dopaminergic neurons resulting from the synergistic effects of rotenone $(5 \mathrm{nM})$ and glutamate $(100 \mu \mathrm{M})$ was further indicated by the calculation of a differential toxicity ratio (DTR) as described in Materials and Methods. A DTR value of 0 indicates that mesencephalic dopaminergic and GABAergic neurons are similarly destroyed, whereas a value of 1 reveals that the treatment destroys all dopaminergic neurons but only partially destroys the GABAergic neurons. Indeed, the combined exposure of the cells to rotenone (5 nM) and glutamate $(100 \mu \mathrm{M})$ led to a DTR of 0.63 , while DTR values of 0.11 and 0.13 were found when both populations were exposed, respectively, to rotenone or glutamate alone (Fig. $1 A$, Table 1).

Complementary experiments were performed in order to ensure that the estimations of the residual uptakes of ${ }^{3} \mathrm{H}-\mathrm{DA}$ and ${ }^{3} \mathrm{H}$-GABA were reliable indices of survival of the dopaminergic and GABAergic neurons, respectively. For this purpose, the 

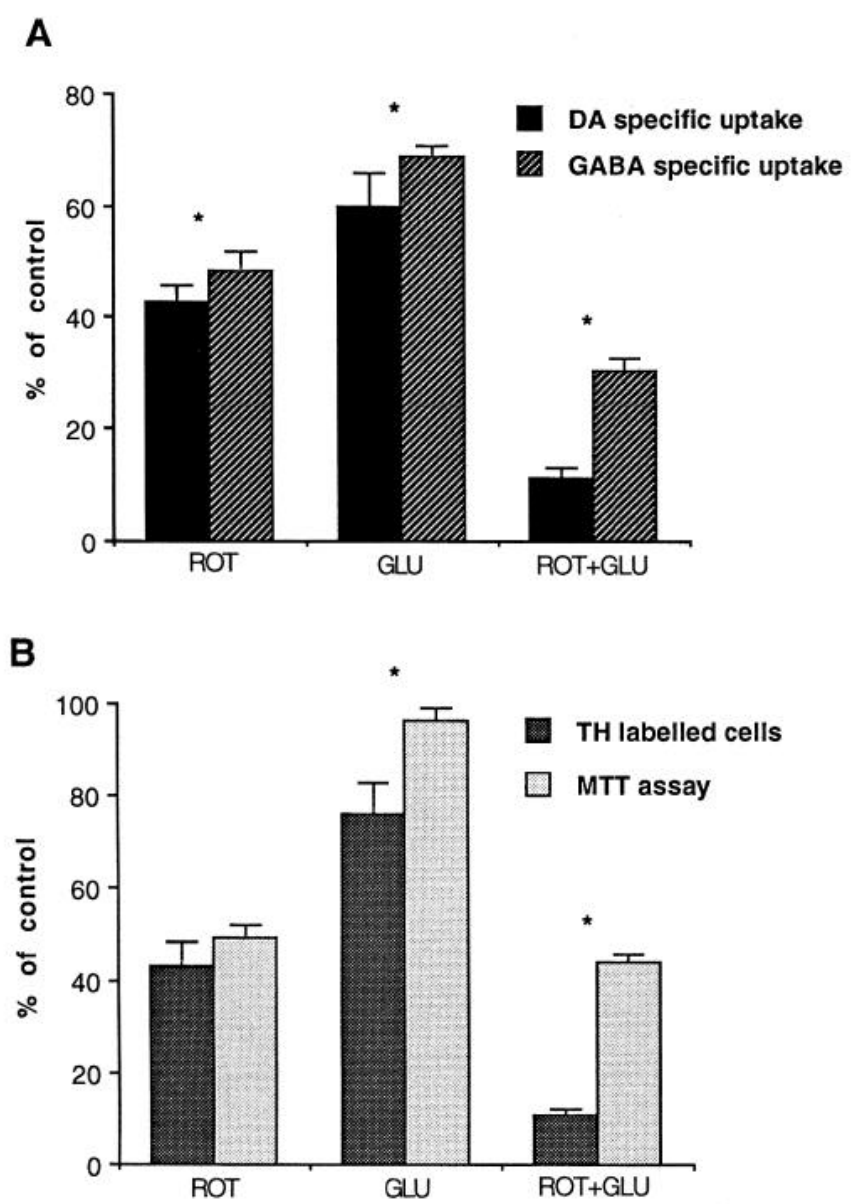

Figure 1. Selective toxicity toward dopaminergic neurons is induced by the synergistic effects of glutamate and an impairment of the energetic metabolism. Mesencephalic neuronal cultures were exposed to 5 nM rotenone $(R O T)$ for $6 \mathrm{hr}$, or to $100 \mu \mathrm{M}$ glutamate $(G L U)$ for $1 \mathrm{hr}$, or to $5 \mathrm{~nm}$ rotenone for $6 \mathrm{hr}$ followed by $100 \mu \mathrm{M}$ glutamate for $1 \mathrm{hr}$. $A$, After a $16 \mathrm{hr}$ recovery period, toxicity toward dopaminergic and GABAergic neurons was determined by measuring their residual specific neurotransmitter uptake. Nonspecific uptake values were determined in the presence of $500 \mu \mathrm{M}$ nipecotic acid or $1 \mu \mathrm{M}$ benztropine. Data are expressed as percentages of control values. Each value is the mean \pm SEM of data obtained from three to nine independent experiments performed in triplicate or quadruplicate. $B$, After a $16 \mathrm{hr}$ recovery period, the survival of the whole mesencephalic neuronal preparation and of the dopaminergic neurons were estimated using the methylthiazolyl tetrazolium-based colorimetric assay (MTT) and TH-immunolabeled cell counting, respectively. Data are expressed in percentages of control values. Each value is the mean \pm SEM of four independent experiments performed in quadruplicate. ${ }^{*} p<0.001$ (Student's $t$ test).

MTT test (Denizot and Lang, 1986) and immunocytochemistry with an anti-tyrosine hydroxylase antibody were used. The MTT test reflects the overall viability of the culture, containing mainly GABAergic neurons, while immunocytochemistry for tyrosine hydroxylase allows the evaluation of the number of dopaminergic neurons. Confirming the results obtained by measuring the residual uptakes of ${ }^{3} \mathrm{H}-\mathrm{DA}$ and ${ }^{3} \mathrm{H}-\mathrm{GABA}$, the data provided by the MTT test and the anti-tyrosine hydroxylase immunocytochemistry further indicated that the dopaminergic neurons were much more vulnerable ( $90 \%$ destruction) than the total neuronal population (mainly GABAergic neurons) (56\% destruction) to the sequential exposure to rotenone and glutamate (Fig. 1B). Still in good agreement with results of specific uptake

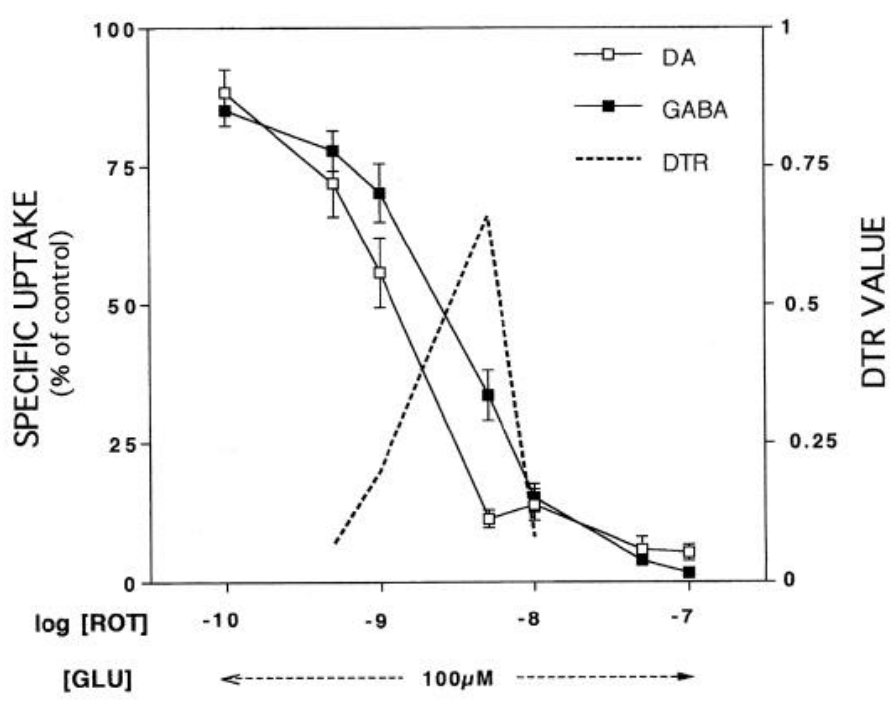

Figure 2. Selective toxic effect of glutamate on mesencephalic neurons pretreated with various concentrations of rotenone. Mesencephalic neuronal cultures were exposed to various concentrations of rotenone $(R O T)$ for $6 \mathrm{hr}$ followed by $100 \mu \mathrm{M}$ glutamate $(G L U)$ for $1 \mathrm{hr}$. After a $16 \mathrm{hr}$ recovery period, toxicity toward dopaminergic ( $\square$ ) and GABAergic neurons $(\square)$ was determined by measuring the residual specific uptake of dopamine and GABA. Nonspecific uptake values were determined in the presence of $500 \mu \mathrm{M}$ nipecotic acid or $1 \mu \mathrm{M}$ benztropine. The DTR value (see Materials and Methods) reached a peak for $5 \mathrm{~nm}$ rotenone. Each value is the mean \pm SEM of three independent experiments performed in quadruplicate.

measurements, no marked differential toxic effect was observed with either $5 \mathrm{~nm}$ rotenone or $100 \mu \mathrm{M}$ glutamate alone (Fig. $1 B$ ).

\section{Effects of rotenone and glutamate on mesencephalic serotoninergic and striatal cholinergic and GABAergic neurons}

The toxic effects of glutamate $(100 \mu \mathrm{M})$ and rotenone $(5 \mathrm{nM})$, alone or in combination, were also investigated on mesencephalic serotoninergic neurons and on striatal cholinergic and GABAergic neurons by measuring the residual specific uptake of ${ }^{3} \mathrm{H}-5-\mathrm{HT},{ }^{3} \mathrm{H}-\mathrm{choline}$, and ${ }^{3} \mathrm{H}-\mathrm{GABA}$, respectively.

The exposure of cells to either rotenone or glutamate affected to a similar extent these different neuronal populations, the neurotoxic effect of rotenone being more pronounced than that of glutamate in agreement with results obtained on mesencephalic GABAergic and dopaminergic neurons (Fig. 1A, Table 2). However, following sequential exposure to rotenone and glutamate, the residual ${ }^{3} \mathrm{H}-5-\mathrm{HT}$ uptake was particularly low $(13.1 \pm 3 \%$ of control) when compared to that of ${ }^{3} \mathrm{H}-\mathrm{GABA}$ in mesencephalic and striatal cultures $(33.6 \pm 4.5 \%$ and $30 \pm 3 \%$, respectively), indicating the existence of a high vulnerability of serotoninergic neurons to this combined treatment, as observed for mesencephalic dopaminergic neurons. The cholinergic interneurons were even less affected than the GABAergic neurons since the residual uptake of ${ }^{3} \mathrm{H}$-choline in striatal cultures was identical after exposure to rotenone alone or to the sequential application of rotenone and glutamate (Table 2).

The selective toxicity toward dopaminergic neurons induced by rotenone and glutamate involves the activation of both NMDA and AMPA receptors

Toxicity experiments were performed in the presence of either the specific antagonists or agonists of the ionotropic glutamate 
Table 1. Glutamate-selective toxicity toward dopaminergic neurons involves activation of both NMDA and AMPA receptors in rotenone-pretreated mesencephalic neurons

\begin{tabular}{|c|c|c|c|}
\hline \multirow[b]{2}{*}{ Compound } & \multicolumn{2}{|c|}{ Specific uptake ( $\%$ of control) } & \multirow[b]{2}{*}{ DTR } \\
\hline & Dopamine & GABA & \\
\hline ROT & $43 \pm 2.8(25)$ & $48.5 \pm 3$ & 0.11 \\
\hline GLU & $59.9 \pm 5.9(9)$ & $69 \pm 1.8(9)$ & 0.13 \\
\hline ROT + GLU & $11.3 \pm 1.6(33)$ & $30.4 \pm 2.2(33)$ & $0.63 *$ \\
\hline ROT + NMDA & $36.7 \pm 4.2(7)$ & $51.3 \pm 5.5(6)$ & 0.28 \\
\hline ROT + AMPA & $21.9 \pm 3(9)$ & $47 \pm 3 \quad(9)$ & $0.53^{*}$ \\
\hline $\mathrm{ROT}+\mathrm{NMDA}+\mathrm{AMPA}$ & $8.6 \pm 1.4(19)$ & $26.7 \pm 2.7(19)$ & $0.68 *$ \\
\hline $\mathrm{ROT}+\mathrm{GLU}+\mathrm{APV}$ & $32.1 \pm 6.4(7)$ & $38.5 \pm 9.6(7)$ & $0.16 * *$ \\
\hline $\mathrm{ROT}+\mathrm{GLU}+\mathrm{MK} 801$ & $31 \pm 2.3(13)$ & $42.3 \pm 3.7(13)$ & $0.21 * *$ \\
\hline ROT + GLU + DNQX & $28.1 \pm 3.2(11)$ & $27 \pm 1.7(11)$ & $-0.04 * *$ \\
\hline $\mathrm{ROT}+\mathrm{AMPA}+\mathrm{MK} 801$ & $37.4 \pm 7.3(12)$ & $43.5 \pm 9 \quad(7)$ & $0.14^{* *}$ \\
\hline
\end{tabular}

The addition of $1 \mu \mathrm{M}$ MK801 and $500 \mu \mathrm{M}$ APV, specific antagonists of the glutamate NMDA receptor, or $50 \mu \mathrm{M}$ DNQX, a specific AMPA receptor antagonist, 5 min prior to the treatment with glutamate strongly decreased the DTR value (see Materials and Methods). The combined exposure to $200 \mu M$ NMDA and 100 $\mu \mathrm{M}$ AMPA resulted in a DTR value equivalent to that obtained in the presence of glutamate. Data are expressed in percentages of control values. Number of specific neurotransmitter uptake measurements is indicated in brackets.

${ }^{*} p<0.001$; DTR value is significantly different from that obtained with rotenone alone (Student's $t$ test). In the table, the SEM of DTR values were omitted for simplicity.

${ }^{* *} p<0.001 ;$ DTR value is significantly different from that obtained in the presence of rotenone and glutamate (Student's $t$ test).

receptors in order to identify the glutamate receptor type involved in the selective toxicity of glutamate- on rotenone-pretreated dopaminergic neurons. Antagonists were added $5 \mathrm{~min}$ prior to the glutamate treatment while agonists were applied for $1 \mathrm{hr}$ (as for glutamate) after the $6 \mathrm{hr}$ exposure to rotenone. Results were expressed using the DTR index as previously described.

As shown in Table 1, AMPA and NMDA receptor antagonists reduced the toxicity induced by glutamate on rotenone-pretreated dopaminergic neurons. Indeed, at the single concentration used, not only DNQX $(50 \mu \mathrm{M})$, the AMPA antagonist, but also APV $(500 \mu \mathrm{M})$ or MK801 $(1 \mu \mathrm{M})$, the competitive and noncompetitive NMDA antagonists, had a much higher protective effect on dopaminergic neurons than on GABAergic neurons toward the toxic action of glutamate. In all cases the DTR value was markedly decreased when compared to that found in the absence of the antagonist (0.63) (Table 1). AMPA alone had a surprising preferential toxicity on rotenone-pretreated dopaminergic neurons since the DTR value (0.53) was only slightly lower than that found with glutamate $(0.63)$. This was due to the presence of contaminating glutamate in the culture medium (Driscoll et al., 1993; I. Marey-Sempel, M. Gelman, and M. Lévi-Strauss, unpublished observations), since MK801 markedly reduced this preferential toxicity of AMPA toward dopaminergic neurons (DTR of 0.14). As expected, due to the $\mathrm{Mg}^{2+}$ block, NMDA alone $(200 \mu \mathrm{M})$ had little neurotoxic effect on rotenone-pretreated neurons (Table 1). When the $\mathrm{Mg}^{2+}$ block of NMDA receptors was eliminated by the combined application of AMPA or by the removal of $\mathrm{Mg}^{2+}$ ions during the NMDA application, NMDA induced a particularly pronounced differential toxic effect on dopaminergic neurons (Table 3). Indeed, in both cases the DTR values were particularly elevated $(0.68$ and 0.79 , respectively) in the range of that found with glutamate $(0.63)$ (Tables 1,3 ).

\section{NMDA-selective neurotoxicity on rotenone-pretreated dopaminergic neurons depends critically on the presence of an extracellular physiological sodium concentration}

Using mouse mesencephalic slices, Johnson et al. (1992) have reported that the NMDA-induced burst firing of dopaminergic neurons depends exclusively upon sodium influx through NMDA-gated channels. The influx of this cation could therefore be involved in the high vulnerability to NMDA receptor stim-

Table 2. Effects of rotenone and glutamate on various neuronal populations

\begin{tabular}{|c|c|c|c|c|}
\hline \multirow[b]{3}{*}{ Compound } & \multicolumn{4}{|c|}{ Specific uptake (\% of control) } \\
\hline & \multicolumn{2}{|c|}{ Mesencephalon } & \multicolumn{2}{|l|}{ Striatum } \\
\hline & GABA & 5-HT & GABA & Choline \\
\hline ROT & $48.5 \pm 3$ & $33.6 \pm 8.6$ & $34.9 \pm 2.5$ & $39.3 \pm 3.5$ \\
\hline GLU & $69 \pm 1.8$ & $82 \pm 5.8$ & $89.2 \pm 2.5$ & $76.5 \pm 4.7$ \\
\hline $\mathrm{ROT}+\mathrm{GLU}$ & $33.6 \pm 4.5^{*}(69 \%)$ & $13.1 \pm 3^{*}(39 \%)$ & $30 \pm 3^{*}(86 \%)$ & $42.7 \pm 2.6(108 \%)$ \\
\hline
\end{tabular}

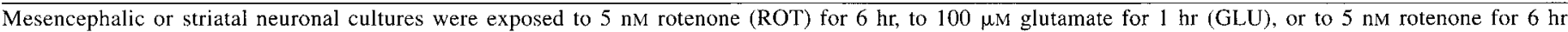

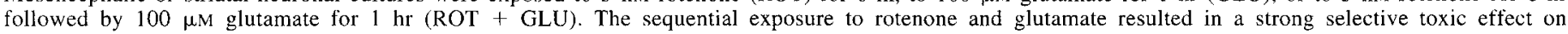

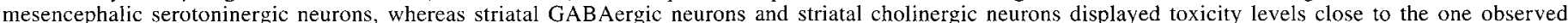

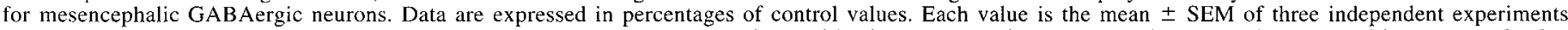

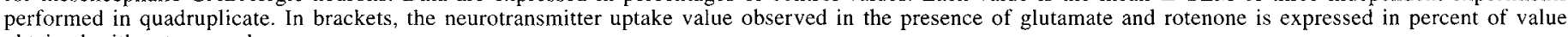
obtained with rotenone alone.

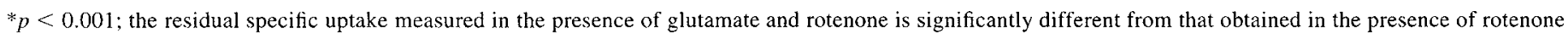
alone (Student's $t$ test). 
Table 3. Comparison of the differential toxic effect induced by $200 \mu \mathrm{M}$ NMDA in the presence or absence of Mg ${ }^{2+}$ ions: role of AMPA receptor activation

\begin{tabular}{|c|c|c|c|c|c|c|}
\hline \multirow[b]{3}{*}{ Compound } & \multicolumn{3}{|l|}{$-\mathrm{Mg}^{21}$} & \multicolumn{3}{|l|}{$+\mathrm{Mg}^{2+}$} \\
\hline & \multicolumn{2}{|c|}{ Specific uptake (\% of control) } & \multirow[b]{2}{*}{ DTR } & \multicolumn{2}{|c|}{ Specific uptake (\% of control) } & \multirow[b]{2}{*}{ DTR } \\
\hline & Dopamine & GABA & & Dopamine & GABA & \\
\hline ROT & $45.9 \pm 3.6(20)$ & $57.7 \pm 5.8(15)$ & 0.2 & $38.4 \pm 2$ & $45 \pm 7 \quad(7)$ & 0.15 \\
\hline ROT + NMDA & $7.8 \pm 1.3(20)$ & $36.4 \pm 3.6(20)$ & $0.79 *$ & $39.5 \pm 4$ & $40 \pm 2 \quad(12)$ & 0.01 \\
\hline $\mathrm{ROT}+\mathrm{NMDA}+\mathrm{AMPA}$ & nd & nd & nd & $16 \pm 2.9(11)$ & $31.2 \pm 2.7(11)$ & $0.49^{*}$ \\
\hline
\end{tabular}

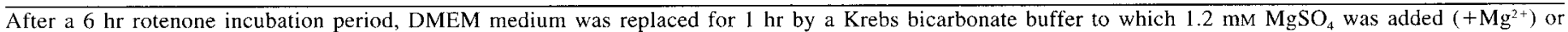

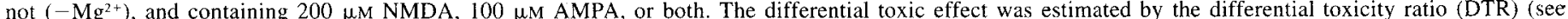

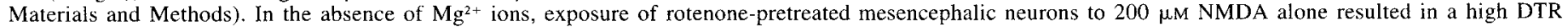

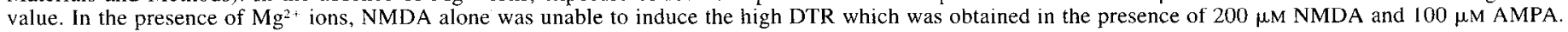
Number of specific uptake measurements is indicated between brackets.

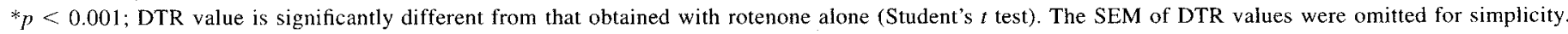

ulation displayed by the rotenone-pretreated dopaminergic neurons. When the extracellular sodium concentration was reduced from $150.3 \mathrm{~mm}$ to $26.3 \mathrm{~mm}$, using choline chloride in place of sodium chloride, the selective toxic effect toward dopaminergic neurons was strongly reduced (DTR of 0.79 in the presence of sodium vs DTR of 0.30 in the presence of choline) (Fig. 3). Choline substitution had a weak effect on the toxicity induced by rotenone alone on dopaminergic and GABAergic neurons (DTR of 0.20 in the presence of sodium vs DTR of 0.29 in the presence of choline). While the NMDA-selective toxic effect toward dopaminergic neurons was strongly reduced by partial

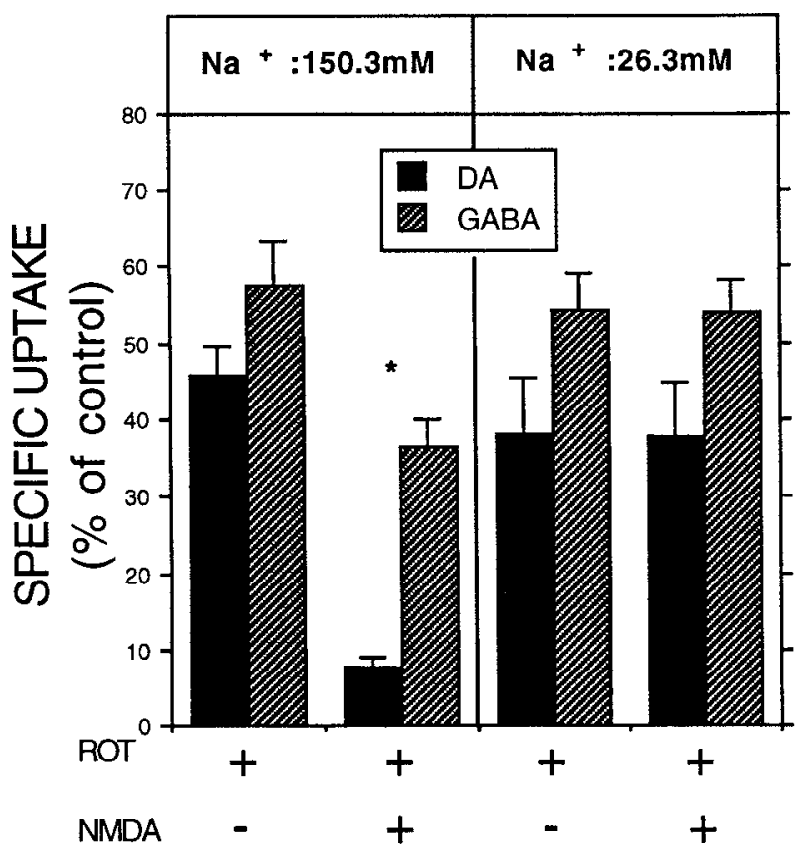

Figure 3. Selective toxicity of NMDA toward dopaminergic neurons in rotenone-pretreated mesencephalic preparation depends critically on extracellular sodium concentration. After a $6 \mathrm{hr}$ incubation period with 5 nM rotenone $(R O T)$, mesencephalic neuronal cultures were incubated in a Krebs bicarbonate buffer to which $200 \mu \mathrm{M}$ NMDA was added or not. When sodium chloride was reduced from $150.3 \mathrm{mM}($ left $)$ to 26.3 $\mathrm{mM}$ (right), using choline chloride in place of sodium chloride, selective NMDA toxicity on dopaminergic neurons was no longer observed. Data are expressed in percentages of control values. Each value is the mean \pm SEM of data obtained from two to five experiments performed in quadruplicate. ${ }^{*} p<0.0001$ (Student's $t$ test); specific dopamine uptake was significantly different from GABA-specific uptake. sodium replacement, it was not blocked by the addition of $1 \mu \mathrm{M}$ TTX.

\section{Discussion}

The strong synergistic effects of rotenone and glutamate, which when used together much more efficiently destroy dopaminergic neurons than GABAergic neurons, stand in sharp contrast to the lack of selectivity toward dopaminergic neurons of the toxicity induced by either of these two compounds. Interestingly, a lack of selectivity toward dopaminergic neurons of the neurotoxicity induced by various concentrations of glutamate has also been reported by Kikuchi and Kim (1993), who have sought such a differential toxicity in order to investigate the role of excitatory amino acids in the death of dopaminergic neurons. The lack of selectivity toward dopaminergic neurons of the neurotoxicity induced by rotenone contrasts with our previous results demonstrating a particularly high sensitivity to rotenone of the specific uptake process of DA (Marey-Semper et al., 1993). This discrepancy can be explained not only by variations in the concentrations of rotenone and incubation time $(10 \mu \mathrm{M}, 60 \mathrm{~min}$ in sensitivity measurements vs $5 \mathrm{nM}, 360 \mathrm{~min}$ in toxicity measurements), but also by the presence or absence of glutamate in the culture medium used in the sensitivity or toxicity experiments, respectively.

In order to rule out the hypothesis of a particular resistance of mesencephalic GABAergic neurons to the synergistic actions of rotenone and glutamate, the vulnerability of other neuronal types was also investigated. Striatal cholinergic and GABAcrgic neurons were, in fact, found to display the same weak vulnerability to the combined treatment with rotenone and glutamate as the mesencephalic GABAergic neurons. One should note that the striatal cholinergic neurons were found to be even more resistant to neurotoxicity than the striatal GABAergic neurons, as previously shown in many different systems (Davies and Beardsall, 1992; Henshaw et al., 1994). Interestingly, the high sensitivity of serotoninergic mesencephalic neurons to the combined action of these compounds can be related to the natural vulnerability of these neurons, which are also affected in the course of Parkinson's disease (Scatton et al., 1983). Although the degeneration of nigrostriatal dopaminergic neurons is the main physiopathological feature of Parkinson's disease, other neuronal types are also destroyed. Parkinson's disease indeed appears to affect ascending noradrenergic, cholinergic, and serotoninergic ncurons whose cell bodies are also located in the mesencephalon. Unfortunately, we were unable to test the vulnerability of 
mesencephalic cholinergic and noradrenergic neuronal populations due to the prohibitive levels of nonspecific uptake that we measured for these two neurotransmitters in mesencephalic cultures (data not shown).

Although the selective toxicity induced by glutamate toward rotenone-pretreated dopaminergic neurons is abolished in the presence of NMDA receptor antagonists, the combined activation of AMPA and NMDA receptors is, however, necessary to reproduce it. The most likely explanation for the requirement of both AMPA and NMDA receptor stimulation is that the AMPA receptor activation could enable the relief of the voltage-dependent $\mathrm{Mg}^{2+}$ block of the NMDA receptor channel (Nowak et al., 1984; for review, see Collingridge and Singer, 1990). Accordingly, in a Krebs-bicarbonate buffer deprived of $\mathrm{Mg}^{2+}$ ions, the addition of NMDA alone induced a high selective toxicity toward dopaminergic neurons that was abolished in the presence of $\mathrm{Mg}^{2+}$ ions. It is important to note that the selective toxic effect of AMPA on dopaminergic neurons observed in a DMEM medium disappears in the presence of MK801, a specific NMDA receptor antagonist. This observation suggests that the low level of glutamate present in the theoretically glutamate-free DMEM medium estimated at $4.5 \mu \mathrm{M}$ (Driscoll et al., 1993; Marey-Semper, Gelman, and Lévi-Strauss, unpublished observations) is sufficient to activate the NMDA receptor. Altogether, these results indicate that, following impairment of the energetic metabolism, NMDA receptor activation is directly responsible for the selective toxicity induced by glutamate on dopaminergic neurons.

This involvement of the NMDA receptors in the high vulnerability of the dopaminergic neurons to the synergistic actions of rotenone and glutamate fits very well with the electrophysiological characterization of the NMDA-induced burst firing of dopaminergic neurons from midbrain slices (Johnson et al., 1992). Indccd, Johnson et al. (1992) reported that the activation of NMDA receptors leads, in mesencephalic dopaminergic neurons, to a particularly high influx of sodium. They proposed that the elevated intracellular sodium concentration induced by NMDA receptor activation in these neurons could give rise to an increased activity of the energy-consuming $\mathrm{Na}^{+} / \mathrm{K}^{+}$ATPase, whose outward current would be involved in the burst firing mechanism. This hypothesis is strengthened by our results indicating that the presence of a physiological extracellular sodium concentration is necessary for the selective neurotoxicity induced by NMDA on rotenone-pretreated dopaminergic neurons (Fig. 3). Moreover, the absence of protective effects of TTX toward dopaminergic neurons indicates that the contribution of voltage-dependent sodium channels to the NMDA-induced, sodium-dependent neurotoxicity is very small (data not shown). One may suggest that after an energetic impairment such as the one induced by rotenone, the preferential death of dopaminergic neurons could be due to the high energy consumption of their $\mathrm{Na}^{+} / \mathrm{K}^{+}$ATPase induced by their particularly elevated NMDAdependent sodium influx. A sodium-dependent, TTX-insensitive component of glutamate neurotoxicity has also been described by Choi (1987) in cultured cortical neurons. Another possible consequence of this excessive entry of $\mathrm{Na}^{+}$through the NMDA receptor may be the potentiation of the $\mathrm{Ca}^{2+}$-induced neurotoxicity due to a reduction of the rate of $\mathrm{Ca}^{2+}$ extrusion by the membrane $\mathrm{Na}^{+} / \mathrm{Ca}^{2+}$ exchanger (Kiedrowski, 1994).

Our model provides an explanation for the protective effects of NMDA receptor antagonists against the dopaminergic degeneration induced by metamphetamine or $\mathrm{MPP}^{+}$(Sonsalla et al., 1989; Turski et al., 1991), whose neurotoxicity mechanisms are thought to involve an impairment of the energetic metabolism (Chan et al., 1991, 1994). Our results could also explain the natural vulnerability of the nigrostriatal dopaminergic neurons that are selectively destroyed in the course of Parkinson's disease in which mitochondrial abnormalities have been recently described in nonaffected tissues such as platelets and muscle (for review, see Di Mauro, 1993). The activation of the NMDA receptor by endogenous glutamate could render dopaninergic neurons much more sensitive than other cell types to the ubiquitous mild ATP depletion which should be induced by these mitochondrial abnormalities.

\section{References}

Bates TE, Heales SJR, Davis SEC, Boakye P, Clark JB (1994) Effects of 1-methyl-4-phenylpyridinium on isolated rat brain mitochondria: evidence for a primary involvement of energy depletion. $\mathbf{J}$ Neurochem 63:640-648.

Chamak B, Prochiantz A (1989) Influence of extracellular matrix proteins on the expression of neuronal polarity. Development 106:483491.

Chan P, DeLanney LE, Irvin I, Langston JW, Di Monte DA (1991) Rapid ATP loss caused by 1-methyl-4-phenyl-1,2,3,6-tetrahydropyridine in mouse brain. $J$ Neurochem 57:348-351.

Chan P, Di Monte DA, Luo J-J, DeLanney LE, Irvin I, Langston JW (1994) Rapid ATP loss caused by metamphetamine in the mouse striatum: relationship between energy impairment and dopaminergic neurotoxicity. J Neurochem 62:2484-2487.

Choi DW (1987) Ionic dependence of glutamate neurotoxicity. J Neurosci 7:369-379.

Collingridge GL, Singer W (1990) Excitatory amino acid receptors and synaptic plasticity. Trends Pharmacol Sci 11:290-296.

Coyle JT, Snyder S (1969) Antiparkinsonism drugs: inhibition of DA uptake in the corpus striatum as a possible mechanism of action. Science 166:899-901.

Davies SW, Beardsall K (1992) Nerve growth factor selectively prevents excitotoxin induced degeneration of striatal cholinergic neurones. Neurosci Lett 140:161-164.

Davis CG, Williams AC, Markey SP, Ebert MH, Caine ED, Reichert CM, Kopin IJ (1979) Chronic parkinsonism secondary to intravenous injection of meperidine analogues. Psychiatr Res 1:249-254.

Denizot F, Lang R (1986) Rapid colorimetric assay for cell growth and survival. Modification of the tetrazolium dye procedure giving improved sensitivity and reliability. J Immunol Methods 89:271-277.

Di Mauro S (1993) Mitochondrial involvement in Parkinson's disease: the controversy continues. Neurology 43:2170-2172.

Driscoll BF, Law MJ, Crane AM (1991) Cell damage associated with changing the medium of mesencephalic cultures in serum-free medium is mediated via $N$-methyl-D-aspartate receptor. J Neurochem 56 : 1201-1206

Ernster L, Dallner G, Azzone GF (1963) Differential effects of rotenone and amytal on mitochondrial electron and energy transfer. $J$ Biol Chem 238:1124-1131.

Heikkila RE, Hess A, Duvoisin RC (1984) Dopaminergic neurotoxicity of 1-methyl-4-phenyl-1,2,3,6-tetrahydropyridine in mice. Science 22 : 151-153.

Heikkila RE, Nicklas WJ, Vyas I, Duvoisin RC (1985) Dopaminergic toxicity of rotenone and 1-methyl-4-phenylpyridinium ion after their stereotaxic administration to rats: implication for the mechanism of 1-methyl-4-phenyl-1,2,3,6-tetrahydropyridine toxicity. Neurosci Lett 62:389-394.

Henshaw R, Jenkins BG, Schulz JB, Ferrante RJ, Kowall NW, Rosen BR, Flint Beal M (1994) Malonate produces striatal lesions by indirect NMDA receptor activation. Brain Res 647:161-166.

Javaiid JI, Perel JM, Davis JM (1979) Inhibition of biogenic amines uptake by imipramine, desimipramine, $2-\mathrm{OH}$-imipramine and $2-\mathrm{OH}-$ desimipramine. Life Sci 24:21-28.

Johnson SW, Seutin V, North RA (1992) Burst-firing in dopaminergic neurons induced by $N$-methyl-D-aspartate: role of electrogenic sodium pump. Science 258:665-667.

Kiedrowski I, Brooker G, Costa E, Wroblewski JT (1994) Glutamate impairs neuronal calcium extrusion while reducing sodium gradient. Neuron 12:295-300. 
Kikuchi S, Sim SU (1993) Glutamate neurotoxicity in mesencephalic dopaminergic neurons culture. J Neurosci Res 36:558-569.

Langston JW, Forno LS, Rebert CS, Irwin I (1984) Selective nigral toxicity after systemic administration of 1-methyl-4-phenyl-1,2,3,6tetrahydropyridine (MPTP) in the squirrel monkey. Brain Res 292: 390-394.

Marey-Semper I, Gelman M, Lévi-Strauss M (1993) The high sensitivity to rotenone of striatal dopamine uptake suggests the existence of a constitutive metabolic deficiency in dopaminergic ncurons from the substantia nigra. Eur J Neurosci 5:1029-1034.

Novelli A, Reilly JA, Lysko PG, Hennebery RC (1988) Glutamate becomes neurotoxic via the $N$-methyl-D-aspartate receptor when intracellular energy levels are reduced. Brain Res 451:205-212.

Nowak L, Bregestovski P, Ascher P, Herbet A, Prochiantz A (1984) Magnesium gates glutamate-activated channels in mouse central neurones. Nature 307:462-465.

Radian R, Ottersen OP, Storm Mathisen J, Castel M, Kanner BI (1990) Immunocytochemical localization of the GABA transporter in rat brain. J Neurosci 10:1319-1330.
Scatton B, Javoy-Agid F, Rouquier L, Dubois B, Agid Y (1983) Reduction of cortical dopamine, noradrenaline, serotonin and their metabolites in Parkinson's disease. Brain Res 275:321-328.

Schousboe A, Thorbek P, Hertz L, Krogsggaard-Larsen P (19/9) Effects of GABA analogues of restricted conformation on GABA transport in astrocytes and brain cortex slices and on GABA receptor binding. J Neurochem 33:181-189.

Sonsalla PK, Nicklas WJ, Heikkila RE (1989) Role for excitatory amino acids in metamphetamine-induced nigrostriatal dopaminergic toxicity. Science 243:398-400.

Turski L, Bressler K, Rettig KJ, Löschmann PA, Wachtel H (1991) Protection of substantia nigra from $\mathrm{MPP}^{+}$neurotoxicity by $N$-methylD-aspartate antagonists. Nature 39:414-418.

Zeevalk GD, Nicklas WJ (1991) Mechanisms underlying initiation of. excitotoxicity associated with metabolic inhibition. J Pharmacol Exp Ther 257:870-878.

Zeevalk GD, Den-Yellin E, Nicklas WJ (1995) NMDA receptor involvement in toxicity to dopamine neurons in vitro caused by the succinate dehydrogenase inhibitor 3-nitropropionic acid. J Neurochem 64:455-458. 